\title{
Essais
}

ESSAIS

Revue interdisciplinaire d'Humanités

17 | 2021

Quels lieux pour les morts?

\section{Tombes et cryptes : entre présence et absence}

Avant-propos

Tombs and crypts: Between presence and absence

Foreword

Éric Benoit

\section{OpenEdition}

Journals

Édition électronique

URL : https://journals.openedition.org/essais/8394

DOI : 10.4000/essais.8394

ISSN : 2276-0970

Éditeur

École doctorale Montaigne Humanités

Édition imprimée

Date de publication : 15 avril 2021

Pagination : 7-15

ISBN : 979-10-97024-09-3

ISSN : $2417-4211$

Référence électronique

Éric Benoit, «Tombes et cryptes : entre présence et absence », Essais [En ligne], 17 | 2021, mis en ligne le 27 avril 2021, consulté le 18 janvier 2023. URL : http://journals.openedition.org/essais/8394 ; DOI : https://doi.org/10.4000/essais.8394 


\section{Tombes et cryptes: \\ entre présence et absence}

\section{Avant-propos}

\section{Éric Benoit}

"C'est ici l'esplanade du souvenir, l'enceinte avec solennité où la chose abolie atteste, parmi ce qui est encore, qu'elle fut. »

Paul Claudel, Connaissance de l'Est, « La Tombe »

Le présent volume, résultat du Colloque Doctoral franco-chinois qui s'est tenu à l'Université Bordeaux Montaigne les 7 et 8 novembre 2019, s'inscrit dans une série d'actions de recherche auxquelles ont contribué, depuis plusieurs années, des enseignants-chercheurs et des doctorants de l'Université Bordeaux Montaigne et de l'Université de Wuhan (Chine). Le fil conducteur thématique en a été porté par une comparaison interculturelle des conceptions de l'espace, et notamment du sacré dans l'espace social. La réflexion se poursuit donc ici en direction de l'espace dévolu aux morts et à la mort en Occident, en Chine, mais aussi ailleurs (Japon, Bénin...). À cette dimension interculturelle s'ajoute une dimension trans-séculaire, puisque les articles qu'on va lire concerneront aussi bien l'Antiquité, le Moyen âge, le dix-neuvième siècle, le vingtième siècle, l'époque contemporaine; et aussi une dimension résolument pluridisciplinaire : anthropologie, sociologie, histoire, géographie, sinologie, philosophie, ainsi que les arts et la littérature, car la réflexion concernera non seulement les espaces réels mais aussi les représentations de ces espaces.

L'idée à partir de laquelle a émergé le sujet de ce travail collectif est la suivante : dans le voisinage des livres d'Édgar Morin (L'Homme et la mort), de Baudrillard (L'Échange symbolique et la mort), et de Jankélévitch (La Mort), les travaux de l'historien Philippe Ariès (Essais sur l'histoire de la mort en Occident du Moyen Âge à nos jours, 1975, et L'Homme devant la mort, 1977) ont montré comment, au cours des derniers siècles, s'est opérée une progressive occultation de la mort en Occident : très présente dans le quotidien médiéval, la mort est peu à peu devenue un tabou, rendue de plus en plus invisible jusqu'à sa relégation actuelle dans les espaces marginaux des hôpitaux et des maisons 
de retraite. La mort reste pourtant bien perceptible, dans les familles, dans les rites religieux, dans l'espace matériel des cimetières, de certains lieux de mémoire, et de divers monuments. Les recherches de Michel Vovelle, notamment dans La Mort et l'occident de 1300 à nos jours' ${ }^{1}$, ont poursuivi la réflexion, en l'orientant vers certains types de lieux comme les cimetières provençaux ${ }^{2}$. Patrick Baudry a ensuite approfondi l'enquête dans le domaine de la sociologie et de l'anthropologie en ouvrant de riches perspectives pluridisciplinaires dans son ouvrage intitulé La Place des morts ${ }^{3}$. La dimension plus visuelle et architecturale des lieux donnés aux mort a été étudiée par Michel Ragon dans L'Espace de la mort. Essai sur l'architecture, la décoration et l'urbanisme funéraires ${ }^{4}$. Et parmi les travaux les plus récents on pourra se reporter au livre imposant et richement illustré de Thomas Lequeur, Le Travail des morts 5 . Nous pouvons poursuivre l'interrogation en nous demandant comment, dans ces lieux, cohabitent le sacré et le laïque, comment s'articulent le public et le privé, l'individuel et le collectif, la monstration et le refoulement, le visible et l'invisible, le désarroi et la recherche de sens, le réel et le symbolique, les mots et les gestes, l'émotion et la ritualisation, l'extériorité et l'intériorité, la parole et le silence, la perte et la hantise, l'encore-là et le ne-plus-ici, la présence et l'absence, la séparation et la réparation, la disparition et la relation, la distance et le lien, les objets et les pensées (ou l'impensable)... autant de dualités qui demeurent en tension, car, si les lieux des morts sont à l'usage des vivants, les vivants ne sont jamais très à l'aise dans leurs relations avec les morts.

La réflexion menée dans ce numéro se centrera donc sur les espaces dévolus à la mort et aux morts dans les sociétés, dans le monde urbain et dans le monde rural, mais aussi dans les représentations, notamment littéraires, que nous avons de ces espaces. Ce besoin de lieux pour les morts est attesté par l'archéologie depuis les époques les plus anciennes de l'humanité6, et des textes comme Antigone (de Sophocle à Anouilh) en témoignent aussi exemplairement ${ }^{7}$.

1 Michel Vovelle, La Mort et l'occident de 1300 à nos jours, Gallimard, 1983.

2 Michel Vovelle, La Ville des morts : essai sur l'imaginaire collectif urbain d'après les cimetières provençaux, 1800-1980, CNRS, 1983.

3 Patrick Baudry, La Place des morts : enjeux et rites, Armand Colin, 1999, et L'Harmattan, 2006.

4 Michel Ragon, L'Espace de la mort. Essai sur l'architecture, la décoration et l'urbanisme funéraires, Albin Michel, 1981, 2013.

5 Thomas Lequeur, Le Travail des morts. Une histoire culturelle des dépouilles mortelles, Gallimard, 2018.

6 Voir par exemple les travaux d'Éric Crubézy, Aux Origines des rites funéraires. Voir, cacher, sacraliser, Éditions Odile Jacob, 2019.

7 Les œuvres d'art peuvent d'ailleurs se constituer comme espaces symboliques substitutifs de tombeaux absents. C'est par exemple le cas du premier mouvement de la Treizième Symphonie de Chostakovitch (1962), qui commémore les victimes du massacre de Juifs ukrainiens commis à Babi Yar par les nazis en 1941. Le poème d'Evgueni Evtouchenko chanté par le chœur de basses commence par ces mots : "À Babi Yar il n'y a pas de monument "... C'est le poème lui-même et l'œuvre musicale, qui se font monument de mémoire pour les assassinés. 
Nous nous ouvrirons donc ici à une approche comparatiste, concernant notamment la structure et les fonctions de ces espaces de la mort dans différentes aires culturelles. Il sera intéressant, par exemple, de comparer avec la Chine l'évolution de la situation des espaces de la mort en Occident. On sait que la culture chinoise traditionnelle (avec ses différentes déclinaisons dans le confucianisme, dans le taoïsme, et dans le bouddhisme) est particulièrement soucieuse de la démarcation entre le monde des morts et le monde des vivants, comme on le voit dans le traité De la mort écrit par Wang Chong au premier siècle de notre ère, ou encore à la fin du XVII siècle dans les contes de Pu Songling qui provoquent un effet d'inquiétude par les transgressions de la frontière entre les deux mondes (et Paul Claudel en 1901 a fondé sa pièce chinoise intitulée Le Repos du septième jour sur la logique de cette limite entre les deux mondes), et cette obsession perdure jusque dans le roman récent de Yu Hua, Le Septième Jour, dont les personnages sont des morts laissés sans sépulture ${ }^{8}$. Mais Anne Cheng nous a rappelé que la pensée chinoise considère aussi la mort comme une transformation du $q i$ de l'être vivant, donc dans la continuité de la vie 9 , et l'article de Marcel Granet intitulé «Le langage de la douleur d'après le rituel funéraire de la Chine classique » (1922) montrait déjà que les rituels confucéens, tels qu'ils se présentent dans le Li ki (Mémoire sur les rites) et dans le $Y i$ li (Livre du cérémonial), visent au rétablissement de l'équilibre social du monde des vivants perturbé par l'événement traumatisant de la mort - le culte des ancêtres permettant d'espérer une influence positive des morts sur les vivants. Alors, comment la visibilité et l'invisibilité de la mort dans l'espace urbain et dans l'espace rural ont-elles évolué depuis la Chine ancienne, dans les transformations de la Chine du XX $X^{\mathrm{e}}$ siècle, et jusqu'à aujourd'hui ${ }^{10}$ ? Concernant la Chine ancienne, la région de Xian par exemple offre dans ses paysages de nombreuses collines qui signalent des vestiges de tombeaux impériaux : pas seulement le célèbre tombeau de l'empereur Qin Shi Huang Di à la fin du III siècle avant notre ère, mais aussi des tombeaux plus récents, de l'époque Tang (VIII ${ }^{\mathrm{e}}-\mathrm{IX}^{\mathrm{e}}$ siècles) ; et n'oublions pas par ailleurs les fameux tombeaux Ming de la région de Pékin. Archéologiquement, certaines régions de la Chine avec ses si nombreux tombeaux font penser à l'Égypte avec ses pyramides et sa Vallée des Rois. Les écrivains occidentaux qui ont voyagé en Chine ont parfois été frappés par les lieux chinois de la mort ; c'est le cas de Claudel, diplomate en Chine de 1895 à 1909, qui évoque plusieurs fois les tombes chinoises en forme d'oméga dans Connaissance de l'Est (1909) ; ou

8 Yu Hua, Le Septième Jour [2013], roman traduit du chinois par Angel Pino et Isabelle Rabut, Actes Sud, 2014.

9 Anne Cheng, Histoire de la pensée chinoise, Seuil, 1999, p. 454.

10 Voir les chapitres consacrés à la Chine dans l'ouvrage collectif dirigé par Natacha AvelineDubach, La Place des morts dans les mégalopoles d'Asie orientale, Éditions Les Indes savantes, 2013. 
encore de Henri Michaux qui, revenant de son voyage en Orient, écrit dans Un Barbare en Asie (1933) : " D'ailleurs, le tiers de la Chine est un cimetière. Mais quel cimetière ! / La campagne chinoise, quand je la vis pour la première fois, m'a été droit au cour. Des tombes, des montagnes entières ou plutôt le flanc de l'une, le côté Est d'une autre, couvertes de tombes " ${ }^{11}$. L'écrivain occidental séjournant en Chine est ainsi confronté non seulement à l'autre monde qu'est une civilisation radicalement étrangère, mais aussi à cet outre-monde sur lequel s'ouvrent les lieux dévolus aux morts.

L'accueil de ce dossier dans la revue Essais se justifie d'abord par les recherches des collègues et doctorants de notre université sur ce sujet, et par les collaborations de recherche que notre université entretient avec des universités chinoises. Mais nous pouvons aussi nous souvenir ici de l'attitude spéculative quasiment expérimentale qui fut celle de Montaigne dans ses Essais : car du fait de la difficulté du rapport des vivants vis-à-vis des morts, chaque civilisation s'essaie à construire sa relation aux morts à travers des lieux inscrits dans l'espace collectif, et, partant, chaque être humain s'essaie à négocier, tant bien que mal, son lien aux disparus qui lui sont restés chers. L'auteur des Essais lui-même, qui nous enseigna "que philosopher, c'est apprendre à mourir " (Livre Premier, chapitre 20), mais pour qui la méditation sur la mort se devait d'être le prélude à un hymne à la vie, n'a-t-il pas tenté de bâtir un tombeau littéraire pour son défunt ami La Boétie ? Les études rassemblées dans ce volume montreront les tentatives, toujours imparfaites, des différences cultures dans la construction de cette relation malaisée des vivants aux morts. Par sa dimension pluridisciplinaire, ce numéro de la revue s'efforcera de rendre compte à la fois des constantes anthropologiques de notre rapport aux morts, et des diversités culturelles qu'implique la difficulté d'une telle relation.

Car le vivant humain, devant ces lieux dévolus aux morts, et notamment à ses morts, aux morts qui lui sont chers, s'interroge : sont-ils là ? Que reste-t-il d'eux? Qui d'autre que moi entend ce que je leur dis ? Dans quelle mesure indécise ces absents sont-ils encore présents? (dans la mesure peut-être d'un " comme si ", "comme si " tu étais encore ici, dans la mesure du " comme si " de la métaphore). Devant ces lieux s'effectue tout un travail de l'imaginaire, de l'élaboration symbolique. Le tombeau est signe (c'est bien le double sens de sèma en grec). Le tombeau fonctionne comme le langage dans sa vertu la plus haute, la plus poétique : pour tenter de signifier cela-même qui excède le langage, pour tenter de dire là-même où le langage défaille, pour tenter de manifester, par le miracle d'un signifiant sans référent, une présence dans

11 Henri Michaux, Un Barbare en Asie, Gallimard, 1933, collection "L'Imaginaire ", 1986, p. 157. 
l'absence. Le tombeau est un signe sans mot mais qui fonctionne comme un mot, un signe sans mot qui se substitue à notre absence de mots, ou qui dans le meilleur des cas suscite en nous les mots qui font être encore un peu quelque chose de celui ou de celle qui n'est plus. Les inscriptions funéraires gravées depuis l'antiquité sur la dalle des tombeaux ont voulu donner à ces mots la durée de la pierre. Ces mots peuvent aussi se faire poème, comme on le voit dans le genre littéraire du tombeau poétique qui s'est développé au $\mathrm{XVI}^{e}$ siècle, et comme on le voit encore exemplairement dans "Demain, dès l'aube... " de Victor Hugo qui s'adresse à sa fille Léopoldine en allant vers la tombe de celle-ci : "Vois-tu, je sais que tu m'attends", " je ne puis demeurer loin de toi plus longtemps", "je mettrai sur ta tombe / Un bouquet de houx vert et de bruyère en fleur " (Les Contemplations, IV, 14). L'édition date ce poème du 3 septembre 1847, la veille du quatrième anniversaire de la mort de Léopoldine. Baudelaire, dans le poème 100 des Fleurs du Mal, aura de semblables méditations : "Les morts, les pauvres morts ont de grandes douleurs ", pense-t-il en évoquant celle qui " dort son sommeil sous une humble pelouse ".

Ce lieu qu'est le tombeau fonctionne aussi comme un étrange miroir différé, comme une sorte de stade du miroir par anticipation : devant cette dalle opaque et muette et sans visage, je suis renvoyé à celui que je serai quand je ne serai plus. Prémonition anthume de ma disparition posthume. Des écrivains ont excellé dans l'élaboration ante-mortem de leur propre tombeau, d'un lieu symbolisant leur mort à venir, leur état de mort dans le futur. On appelle parfois cela un livre. Chateaubriand, Mémoires d'outre-tombe, je vous écris d'un pays lointain, je vous parle d'un lieu qui est celui de l'au-delà, " comme si " j'étais déjà mort, entendez donc ma voix oraculairement venue d'ailleurs et qui pourtant vous parvient déjà et encore de cet au-delà auquel je vous donne mystérieusement accès... "Ce livre doit être lu comme on lirait le livre d'un mort ", dixit Victor Hugo en Préface des Contemplations (1856) : le livre est un tombeau de soi-même, et la voix en acquiert une autorité sacrée, comme transcendantale, dressée dans le ci-gît de son érection paradoxale.

Et moi-même lecteur : lorsque je lis, le plus souvent je lis la parole d'un mort. Nos bibliothèques sont de fantastiques nécropoles, sont de magnifiques monuments de la mort sublimée, et ces petits tombeaux portatifs de l'âme qu'on appelle des livres, nous avons le pouvoir, lorsque nous les lisons, lorsque nous redonnons notre voix vive à leur parole de poussière, d'en opérer la résurrection. "Lazare, veni foras!» : ainsi Maurice Blanchot désignait-il cette résurrection des morts opérée par l'acte de lecture. Seule pâque dans l'impasse absolue.

Au commencement de nos cultures d'êtres humains, je revois cet homme errant, sans lieu, fuyant tout sol à l'est d'Eden, car il vient de percevoir cette voix qui lui a dit : "J'ai entendu les sangs de ton frère qui crient depuis le sol ! ». Cain l'errant s'enfuit de tout sol, sans lieu pour mémorer Abel mort, 
refoulant le mort dans sa "conscience " (Hugo encore) ou plutôt dans son inconscient. $A$ q quelques générations de là je vois Abraham l'aimant acheter un sol pour le tombeau de Sara, pour le besoin de remémorer l'aimée. Et bientôt on annoncera que la résurrection des morts s'inaugurera dans la vallée de Josaphat, et voici les rives abruptes du Cédron, au pied de Jérusalem, se couvrir de tombeaux et devenir une dense nécropole en attente de la fin des temps. Quelques siècles encore, et l'on nous raconte que des disciples effrayés découvrent au matin du Golgotha un tombeau vide, et qu'un ange leur annonce que celui qu'ils cherchaient parmi les morts n'y est plus, allez en Galilée... Corps délocalisé... Il faut peut-être quitter les lieux des morts pour continuer de vivre...

La même chose peut se dire en un autre langage. Poursuivant les hypothèses développées par Freud dans Deuil et mélancolie (1915), Maria Torok et Nicolas Abraham, dans L'Écorce et le noyau ${ }^{12}$, théorisent la "crypte intrapsychique " dans laquelle nous entretenons une relation avec les morts que nous y avons intériorisés. Ils distinguent alors (on remarquera que ces notions reposent sur des métaphores spatiales héritées des topiques freudiennes) l'incorporation du mort dans le psychisme de l'endeuillé (processus statique, morbide, pathologique, mortifère, qui correspond à la mélancolie freudienne et qui peut aller jusqu'à l'autodestruction du sujet dans une pulsion de mort intériorisée : je tue le mort en moi et c'est cela qui me tue) et l'introjection, plus dynamique, qui me resitue et me restitue dans un projet de vie non seulement en me mettant en relation avec ce qu'il y avait de vivant dans celui ou celle que j'ai perdu(e) mais surtout en me permettant de m'en détacher, de m'en désinvestir pour construire de nouveaux liens et de nouveaux projets de vie (ce qui correspond aux étapes du deuil freudien).

Proust nous donne un bel exemple de cette crypte intrapsychique dans le chapitre de la Recherche intitulé "Les intermittences du cour " : "les morts n'existent plus qu'en nous " ${ }^{13}$. Les pages qui suivent retranscrivent un rêve qu'a fait le narrateur à la recherche de sa grand-mère décédée l'année précédente : il descend dans le "monde du sommeil », dans " les artères de la cité souterraine ", dans les " flots noirs " d'" un Léthé intérieur aux sextuples replis " et aborde "sous les porches sombres"; puis (toujours dans le rêve) il se désole : " comme elle doit être malheureuse dans cette petite chambre qu'on a louée pour elle, $[\ldots]$ où elle est toute seule, $[\ldots]$ comme elle doit se sentir seule et abandonnée "; il rencontre alors son père et lui demande : "Où est grandmère ? dis-moi l'adresse ", et se demande à lui-même : " mais où est-ce ?

12 Freud, "Deuil et mélancolie " [ Trauer und Melancholie ", 1915], Métapsychologie, traduction dirigée par Jean Laplanche et J. B. Pontalis, Gallimard, 1968, collection folio-essais, p. 145-171. Maria Torok, et Nicolas Abraham, L'Écorce et le Noyau, Flammarion, 1978.

13 Marcel Proust, Sodome et Gomorrhe [1921], Gallimard, folio classique, 1989, p. 156. 
comment ai-je pu oublier l'adresse $»^{11} \ldots$ Où le rêve se fait symptôme d'une distance que rien ne peut combler, d'une absence qui ne laisse que regret. Les morts sont-ils même encore en nous ? ${ }^{15}$

Il y a sans doute (j'essaie d'en déduire cette constante anthropologique) cette dialectique ou cette double postulation en tout être humain : à la fois, chez les vivants, un besoin de lieux pour entretenir et cultiver la mémoire des morts, et aussi d'autre part la nécessité de se détacher de tout lieu et de tout lien avec les morts afin de continuer pleinement de vivre. N'était-ce pas déjà la leçon du grand poème de Paul Valéry, Le Cimetière Marin : "Ce toit tranquille, où marchent des colombes, / Entre les pins palpite, entre les tombes "... Nous sommes, oui (comment le nier ? comment l'oublier ?), " entre les tombes ", mais la fin du poème nous ouvre à un autre espace : "Le vent se lève !... Il faut tenter de vivre! ", "Courons à l'onde en rejaillir vivant!".

La Première Partie de ce numéro explore le sens des lieux dévolus à la mort. Patrick Baudry réfléchit tout d'abord à l'élaboration symbolique qui se joue dans le lieu du cimetière et dans la ritualité funéraire. En Chine, cette élaboration symbolique du rapport à la mort et aux morts est médiatisée par le confucianisme, le taoïsme, et le bouddhisme, comme l'expliquent WANG Zhan et Wu Changli. Puis l'article de HaN Jing précise la conception du domaine des morts selon le bouddhisme, et notamment les représentations du dieu du monde des morts, Yanluo.

La Deuxième Partie du volume se centre alors sur un certain nombre de lieux de l'espace réel. D'abord en Chine : Chen Jin présente les tombes de la dynastie Tang (VII ${ }^{\mathrm{e}}-\mathrm{IX}^{\mathrm{e}}$ siècles) et la hiérarchie sociale qu'elles reflètent ; et Maylis Bellocq étudie les transformations récentes des cimetières à Shanghai : problèmes posés par le manque d'espace, accentuation de la réforme funéraire, lutte des autorités contre les superstitions, généralisation de la crémation, dispersion des cendres en mer, tendance à la non-conservation des restes, développement des cimetières numériques et des rituels par internet, jusqu'aux évolutions récentes dues à la crise sanitaire de l'épidémie de covid19. Puis au Japon : Fabienne Duteil-Ogata, après avoir rappelé les pratiques funéraires traditionnelles, présente de nouvelles pratiques qui semblent être à l'émergence de nouveaux lieux pour les morts : les « tombes ou ossuaires collectifs aux cultes éternels ", les tombes numériques, le lancer des cendres dans la nature, et les objets funéraires à portée de main. L'article suivant nous transporte en

14 Ibid., p. 157 et 158.

15 L'œuvre peut alors devenir le lieu symbolique où se tisse un lien avec les morts dont l'écriture opère la transposition métaphorique ou cryptée : " un livre est un grand cimetière où sur la plupart des tombes on ne peut plus lire les noms effacés " (Proust, Le Temps retrouvé [1927], Gallimard, folio classique, 1990, p. 210). 
Afrique Noire, au Bénin : Carole Fagadé y explique comment les esclaves du XVIII siècle, au moment de leur départ, anticipaient rituellement leur propre mort auprès de l'Arbre de l'Oubli, et anticipaient le retour post-mortem de leur âme au pays auprès de l'Arbre du Retour ; elle explique aussi l'usage qui encore aujourd'hui est fait de l'Arbre du Retour comme lieu consacré aux morts. Puis Frédérique Drillaut nous fait partager son étude anthropologique de la mise en espace de la toilette mortuaire en soins palliatifs, et la dimension profondément humaine qui s'y révèle. Enfin, retour en Chine avec le sujet particulier du thanatourisme : Vincent Mariet étudie la politique planifiée de fréquentation touristique des lieux qui ont été marqués par la mort lors de la période des guerres civiles et de la Deuxième Guerre mondiale en Chine notamment à Shanghai et à Nanjing, et il se demande : "le souvenir des morts n'est-il pas perverti par la transformation progressive, sous la poussée du tourisme de masse et de considérations politico-économiques, de lieux historiques de souffrances passées en véritables "parcs à thème" guerriers?".

La Troisième Partie du numéro est consacrée à des représentations, notamment littéraires, des lieux dévolus aux morts. Liu Xinyi présente les divers lieux des morts (et des revenants) dans les Contes de Pu Songling (fin du $\mathrm{XVII}$ siècle) : tombes, maisons hantées, monde subaquatique, monde souterrain, monde des Immortels. Les deux articles suivants concernent l'œuvre de Paul Claudel, qui a vécu en Chine entre 1895 et 1909 et dont l'œuvre est ainsi un bon terrain d'analyses pour une perspective interculturelle. CHU Ge étudie la représentation des tombes chinoises dans les poèmes en prose de Connaissance de l'Est, la conception de l'enfer chinois dans le drame Le Repos du septième jour, et les évocations claudéliennes des rites chinois qui révèlent un certain rapport des vivants et des morts et la hantise de la limite entre les deux mondes. À ces éléments, Xu Yi ajoute ceux qui proviennent du séjour de Claudel au Japon de 1922 à 1928, notamment le séisme meurtrier du $1^{\text {er }}$ septembre 1923. Enfin Élodie Galinat prend comme objet d'étude le roman La Chambre des Officiers de Marc Dugain, publié en 1998 : dans le contexte de la représentation de la Première Guerre mondiale, le roman montre (et commémore littérairement) les espaces de la " presque-mort " que sont la tranchée et le lit d'hôpital.

Nul doute que les récents événements épidémiques survenus en Chine puis dans le monde entier apporteront de nouveaux développements à ces questions. À l'heure où j'écris ces lignes au printemps de cette année 2020, on a vu des familles ne pouvoir pas accompagner leurs morts. On a vu les processions de camions blancs réfrigérés. On a vu les longues housses de plastique jetées les unes sur les autres. On a vu les files d'attente pour la recherche des urnes. On a vu les gymnases transformés en morgues géométriquement rangées. On a vu les lignes de boîtes oblongues empilées trois par trois dans des tranchées 
creusées dans l'île de Hart Island au large du Bronx... Et plusieurs articles de ce numéro de la revue Essais prennent déjà en compte ces réalités qui nous sont immédiatement contemporaines.

Des recherches ultérieures pourront se poursuivre sur le socle des travaux donnés dans le présent volume.

Éric Benoit

Université Bordeaux Montaigne

Unité de Recherche TELEM (EA 4195)

Eric.Benoit@u-bordeaux-montaigne.fr

Comité scientifique de ce numéro : Maylis Bellocq, Eric Benoit, Marie De Gandt, Sophie Duval, Delphine Gachet, Violaine Giacomotto, Estelle Mouton-Rovira, Guillaume Muller, Philippe Ortel, Gersende Plissoneau, Catherine Ramond, Fabienne Rihard-Diamond, Jérôme Roger (enseignants-chercheurs à l'Université Bordeaux Montaigne). 